\title{
Especialización vs diversificación del conocimiento tecnológico universitario en las regiones europeas"
}

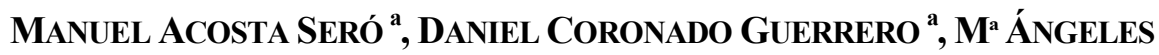 \\ MARTÍNEZ NAVARRO ${ }^{\text {a }}$ \\ a Universidad de Cádiz, Facultad CC.EE., Glorieta Carlos Cano, s/n, 11002 Cádiz. E- \\ mail: manuel.acosta@uca.es, daniel.coronado@uca.es, mariangeles.martinez@uca.es
}

\begin{abstract}
RESUMEN
Este artículo explora la relación entre los patrones regionales de diversificación/especialización del conocimiento tecnológico generado por las universidades en Europa y sus efectos en la producción de nuevos resultados tecnológicos universitarios medidos por las patentes. Nuestra base de datos incluye 4.580 patentes universitarias relativas a 202 regiones de la Unión Europea-15 para el período 1998-2004. Diversas especificaciones econométricas revelan que la estrategia de diversificación de la producción del conocimiento tecnológico universitario favorece la producción de nuevas patentes universitarias en periodos posteriores. La especialización tiene efectos positivos y significativos en los sectores de alta tecnología.
\end{abstract}

Palabras clave: Conocimiento tecnológico universitario, patentes universitarias, especialización tecnológica, diversificación tecnológica.

\section{Specialization versus Diversification of University Technological Knowledge across European Regions}

\begin{abstract}
This paper explores the relationship between regional patterns of university technological diversification/specialization in Europe and its effects on the production of new university technological outcomes as measured by patent counts. Our dataset contains 4,580 university-owned patents related to 202 regions of European Union-15 for the period from 1998 to 2004. Several econometric specifications reveal that a diversification strategy in the production of university technological knowledge favours the generation of subsequent new university patents. Specialization has a positive and significant effect in high-technology sectors.

Keywords: University Technological Knowledge, University Patents, Technological Specialization, Technological Diversification.
\end{abstract}

Clasificación JEL: O31, O32

\footnotetext{
* Los autores agradecen los comentarios de los evaluadores. Esta investigación ha sido financiada por la Junta de Andalucia (P08-SEJ03981).
}

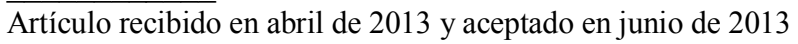

Artículo disponible en versión electrónica en la página www.revista-eea.net, ref. ə-32107 


\section{INTRODUCCIÓN}

Este trabajo se enmarca en los nuevos roles empresariales en los que las universidades han demostrado ser cruciales para el desarrollo económico regional. El término "universidad empresarial" es un concepto amplio sobre el que existe una larga lista de análisis teóricos y empíricos (véase, por ejemplo, la revisión de Yusof y Jain (2010)). La función empresarial de las universidades incluye cinco actividades principales (Etzkowitz y Zhou, 2006): patentar y conceder licencias, consultoría para la industria, creación de nuevas empresas (spin-offs), educación empresarial (formación de los trabajadores de alto nivel) y proporcionar instalaciones para I + D. Este artículo se centra en la primera de esas actividades: el papel de la innovación en las universidades considerada como uno de los principales elementos básicos de una universidad emprendedora (Etzkowitz et al., 2008). Concretamente, el objetivo de este trabajo es explorar la relación entre la especialización/diversificación del conocimiento tecnológico de las universidades y la producción posterior de resultados tecnológicos universitarios (patentes) en las regiones europeas.

Desde el punto de vista político, el análisis de estos aspectos es importante por dos razones. En primer lugar, muchos gobiernos nacionales y regionales tienen capacidad política para guiar hacia qué sectores industriales estratégicos deben orientarse los esfuerzos de producción de tecnología de las universidades. En segundo lugar, promover una estrategia de especialización o de diversificación del conocimiento tecnológico universitario en las regiones puede conducir a resultados diferentes sobre la actividad innovadora.

La metodología se basa en el uso de patentes como medida de la producción de conocimiento tecnológico universitario en las regiones europeas. Los resultados se obtienen a partir de un conjunto de datos de panel integrado por 4.580 patentes propiedad de las instituciones universitarias europeas clasificadas por regiones de la Unión Europea-15 para el período 1998-2004. La metodología empírica consiste en un análisis econométrico para contrastar cómo la especialización/diversificación tecnológica universitaria regional puede afectar a la cantidad de nuevas patentes universitarias.

En este trabajo, se utiliza como unidad de análisis las regiones. La comprensión de cómo está distribuido espacialmente el conocimiento tecnológico universitario y sus características (calidad, especialización,...) facilita la implementación de estrategias regionales que contribuyan a una mejor integración de las universidades en el sistema de innovación regional (Fritsch and Slavtchev, 2007) o en un modelo regional de organización basado en la triple hélice (Etzkowitz and Klofsten, 2005). Desde una perspectiva empírica, la literatura revela que, si bien existe un amplio conocimiento de los efectos positivos de las universidades sobre una serie de variables relevantes para el desarrollo regional (Bercovitz and Feldmann, 2006; Varga, 1998; Audretsch and Lehmann, 2005; 
Woodward et al., 2006; Djokovic and Souitaris, 2008; O'Shea et al., 2008), se sabe poco acerca de los factores que conducen al fortalecimiento de la innovación como parte de las actividades de emprendimiento en las universidades.

Este trabajo contribuye a la literatura con varias aportaciones. En primer lugar, se incluyen nuevos factores que recogen la especialización y la diversificación tecnológica en la función de producción del conocimiento tecnológico universitario (medido por las patentes) considerando las regiones europeas en su conjunto. En segundo lugar, la mayor parte de las regiones europeas tienen autonomía suficiente para organizar sus propios sistemas de innovación; por lo tanto, una mejor comprensión de las características y causas de la generación de nuevo conocimiento tecnológico en las universidades, puede ayudar a definir las políticas que apoyan al desarrollo tecnológico regional.

El artículo está organizado de la siguiente manera. En la sección 2 se presenta un breve resumen de la bibliografía relevante. En la sección 3 se describen los datos. En la sección 4 se presentan varios modelos explicativos de los efectos de la diversificación y la especialización en la producción de patentes universitarias utilizando las regiones europeas como unidad de análisis. Los resultados de las estimaciones econométricas se proporcionan en la sección 5. Por último, en la sección 6 se exponen las principales conclusiones del análisis.

\section{REVISIÓN DE LA LITERATURA}

La revisión de la literatura se organiza en torno a dos cuestiones: a) Cuáles son los principales puntos de vista acerca de la diversificación/especialización tecnológica regional; b) Qué factores son relevantes en la explicación de la producción de conocimiento tecnológico en las universidades.

En cuanto a la primera cuestión, a pesar de que los argumentos en favor de una $u$ otra tesis (especialización o diversificación) tienen una larga tradición en economía urbana, ese debate por el momento no se ha producido en relación a la generación de conocimiento en las universidades. Aunque una revisión completa de la literatura sobre este tema está más allá del alcance de este trabajo, seguidamente se resumen los principales puntos recogidos en varias investigaciones clave (Acs et al., 2002; Carlino et al., 2007; Feldman y Audretsch, 1999; Glaeser et al., 1992).

Por un lado, las externalidades MAR (Marshall-Arrow-Romer) afirman que la concentración de una industria en una ciudad ayuda a la difusión de conocimientos entre las empresas y, por tanto, al crecimiento de la industria y de la ciudad; la especialización en industrias concentradas geográficamente podría por lo tanto estimular el crecimiento. Por otra parte, Jacobs (1969) sostiene que la diversidad de industrias próximas geográficamente promueve en mayor grado la innovación y el crecimiento que la especialización geográfica. 
La investigación empírica sobre el debate especialización/diversificación no contribuye a identificar la mejor estrategia. Algunos trabajos apuntan a una política de especialización para promover la innovación, mientras que otros sugieren que la adopción de medidas de diversificación pueden fomentar las actividades de innovación en el futuro. Feldman and Audrestch (1999) señalan que la diversidad en actividades económicas complementarias que comparten una base científica común es más propicia para la actividad innovadora que la especialización. Kelly and Hageman (1999) concluyen que los clusters de innovación son independientes de la distribución del empleo. Moreno et al. (2006) sostienen, para las regiones europeas, que la innovación de una industria dada está influenciada por el grado de especialización en la misma industria pero no está influenciada por el grado de diversificación del sistema de innovación regional. En la misma línea, Ejermo (2005) obtiene evidencia de que la especialización tecnológica regional afecta al número de patentes solicitadas en el caso de las regiones suecas. Otros estudios relevantes en favor de una u otra estrategia son los de Baptista y Swann (1998); Beaudry y Breschi (2003); Paci y Usai (1999); Shefer y Frenkel (1998); Greunz (2003); Ó Huallacháin y Lee (2011). Beaudry and Schiffauerova (2009) en su revisión de la literatura empírica proporcionan una amplia lista de estudios que favorecen una u otra o ambas tesis. Como señalan las autoras, los resultados son a menudo contradictorios lo que podría explicarse por las diferencias en la intensidad de las fuerzas de aglomeración y por cuestiones metodológicas tales como el nivel de agregación, tipo de industrias y los indicadores utilizados para recoger los diferentes tipos de externalidades.

Trasladando estas ideas al ámbito de la generación de conocimiento por las universidades, se podrían seguir dos estrategias con el fin de fomentar la producción de conocimiento tecnológico universitario en las regiones: por un lado, las externalidades MAR estimulan la especialización tecnológica porque los investigadores de las universidades de una región que trabajan en los mismos temas podrían aprender unos de otros mucho mejor que si trabajan solos debido al flujo de conocimientos en el mismo campo tecnológico; por otro lado, las externalidades Jacobs sugieren que seguir diferentes líneas de trabajo es la mejor manera de aumentar la producción y el stock de conocimientos tecnológico universitario; el flujo de conocimiento entre investigadores de las universidades de la región que trabajan en diferentes campos del conocimiento tecnológico fomentaría nuevas invenciones.

Con respecto a la segunda cuestión, la mayoría de los estudios sobre la producción de conocimiento tecnológico universitario ha utilizado las universidades como unidad de análisis y en el contexto de Estados Unidos. En Europa, este tipo de análisis comenzó con posterioridad, por lo que la evidencia empírica no es tan amplia (Geuna and Nesta, 2006; Baldini et al., 2006; Azagra-Caro et al., 2003, Saragossi and van Pottelsberghe, 2003). Los análisis regionales que 
abordan las causas de la producción de conocimiento tecnológico universitario (medido como patentes) están prácticamente ausentes de la literatura empírica. Sólo Azagra-Caro et al. (2006) para el caso español y Acosta et al. (2009) para las regiones europeas han discutido esta cuestión desde una perspectiva regional. En el primer estudio, los autores construyeron una función de producción de patentes universitarias para identificar los factores que determinan la generación de patentes en 17 comunidades autónomas españolas (NUTS-II) durante 14 años. Los autores obtuvieron en todas las estimaciones una relación positiva significativa entre el número de patentes universitarias y los gastos universitarios en investigación y desarrollo (el gasto universitario en I + D fue el único determinante significativo de las patentes universitarias internacionales). No encontraron evidencia de los efectos de variables tales como el número de investigadores de las universidades, la estructura universitaria, o la estructura de investigación conjunta entre universidades y organizaciones públicas de investigación. El segundo estudio (Acosta et al., 2009) analiza un conjunto de datos de panel de patentes universitarias europeas regionalizados por NUTS II. Sus conclusiones sugieren que las variaciones en gastos universitarios en I + D regional afectan a la actividad de patentar en las regiones, con elasticidades que muestran rendimientos constantes a escala, pero no encontraron evidencia sobre que el potencial industrial de la región fomente la producción de nuevos conocimientos tecnológicos universitarios.

\section{LOS DATOS}

\subsection{La muestra}

La información empleada en este artículo procede de una base de datos con 4.580 patentes universitarias (obtenidas de Derwent Innovation Index) relativas a 378 universidades de 202 regiones europeas (UE-15) abarcando el período 1998-2004. La unidad de análisis son las regiones por lo que se han elegido las unidades territoriales NUTS II de Eurostat, que como se sabe, son unidades territoriales con cierto grado de autoridad administrativa y política. La patente fue asignada a una universidad ( $\mathrm{y}$ en consecuencia a una región) cuando el nombre de la universidad aparecía en la lista de solicitantes, por lo que el nombre de la universidad fue el criterio para la detección de una patente universitaria. Para evitar problemas con el nombre de la Universidad, se examinaron todas aquellas instituciones incluidas en European Indicators, Cyberspace, y ScienceTechnology-Economy-System (EICSTES) Project y en la Worldwide Web of Universities (www.univ.cc, web con enlaces a 7.884 universidades en 190 países). Se han considerado únicamente aquellas patentes propiedad de la universidad lo cual supone que por ejemplo, se excluyen las patentes solicitadas por los investigadores académicos cuando su universidad no aparece en la lista de solicitantes. 
Con el fin de evitar las distorsiones nacionales que surgen de los diferentes requisitos de solicitud de patentes en los distintos países, se incluyeron únicamente las patentes registradas en la Oficina Europea de Patentes. Por otra parte, el mayor coste de una patente nacional en comparación con una patente europea sugiere que se trata de conocimiento tecnológico valioso con potenciales aplicaciones reales.

\subsection{Especialización y diversificación de las patentes universitarias}

Para obtener los índices de especialización/diversificación del conocimiento tecnológico universitario en las diferentes regiones europeas, se clasificaron las patentes de acuerdo con una tabla de concordancia económica desarrollada por Schmoch et al. (2003) entre la Clasificación Internacional de Patentes (CIP) y los 44 sectores industriales. A partir del sector industrial donde se originó la patente, se estableció la relación entre los datos originales de la CIP (a nivel de cuatro dígitos) y los códigos de la Clasificación de Actividades Económicas de la Comunidad Europea (NACE) a nivel de dos dígitos. Se ha tenido en cuenta el primer código CIP de la patente para la generación de la correspondencia entre las subclases de la CIP y los 44 sectores industriales.

En este análisis, se entiende la especialización tecnológica como el grado en el que una región ha concentrado sus esfuerzos para producir patentes mayoritariamente en sectores específicos. Se utiliza como indicador de la especialización regional del conocimiento tecnológico universitario la "ventaja tecnológica revelada" (VTR). La VTR de una región para cada uno de los tres niveles de especialización (alta, media y baja tecnología) se calcula de acuerdo con el indicador tradicional de ventaja comparativa revelada de Balassa (1965):

$$
V T R_{r}=\frac{P_{r j} / \sum_{j} P_{r j}}{\sum_{r} P_{r j} / \sum_{r j} P_{r j}}
$$

donde $\mathrm{P}_{\mathrm{rj}}$ es el número de patentes universitarias de la región $\mathrm{r}$ en el sector $\mathrm{j}$, $\sum_{\mathrm{j}} \mathrm{P}_{\mathrm{rj}}$ es el número total de patentes universitarias de la región $\mathrm{r}, \sum_{\mathrm{r}} \mathrm{P}_{\mathrm{rj}}$ es el número de patentes universitarias de todas las regiones en el sector $\mathrm{j}, \mathrm{y} \sum_{\mathrm{rj}} \mathrm{P}_{\mathrm{rj}}$ es el número total de patentes universitarias (en todas las regiones y todos los sectores).

Como es sabido, valores mayores que uno indican que una región tiene ventaja comparativa en este sector en relación con otras regiones, mientras que los valores inferiores a uno se interpretan en el sentido de desventaja comparativa. Debido a que este índice presenta una distribución asimétrica, para su inclusión en el análisis de regresión se emplea un índice normalizado de la ventaja tecnológica revelada: NVTR $=\left(\mathrm{VTR}^{2}-1\right) /\left(\mathrm{VTR}^{2}+1\right)$ (véanse detalles en Grupp, 1994). Este método tiene la ventaja de la simetría (toma valores entre -1 y +1 ). 
El valor de la ventaja tecnológica revelada se puede interpretar como el grado de especialización regional en un sector $\mathrm{j}$ cuando para ese sector NVTR $>0$.

La distribución de las patentes por niveles tecnológicos (alta, media y baja tecnología) se realizó de acuerdo con la clasificación de la OCDE a partir de la asignación de las patentes a los 44 sectores industriales desarrollados por Schmoch et al. $(2003)^{1}$.

Para medir el grado de diversificación del conocimiento tecnológico generado en las universidades de una región se utiliza el índice de entropía. Este índice mide el grado de diversificación tecnológica teniendo en cuenta la diseminación de las patentes universitarias de una región entre los 44 sectores industriales:

$$
I E_{r}=\sum_{j=1}^{n} T_{r j} \ln \left(\frac{1}{T_{r j}}\right)
$$

donde $T_{\mathrm{rj}}$ es la participación de las patentes universitarias del sector $\mathrm{j}$ en el total de patentes universitarias de la región r. Esta medida considera dos elementos de diversificación tecnológica: el número de sectores en los que una región tiene las patentes y la importancia relativa de esos sectores en el total de patentes en la región. El índice toma el valor 0 para la entropía mínima: un solo sector en la región concentra la producción de patentes universitarias. La entropía es máxima cuando todos los sectores tienen igual participación en las patentes de la región en cuyo caso el valor será igual al "ln ( $\mathrm{n}^{\circ}$ sectores)"; como se están considerando 44 sectores industriales el valor de la entropía máxima es 3,78.

\subsection{Producción de patentes universitarias y la diversificación/especialización tecnológica}

Un examen de los coeficientes de correlación y de las relaciones gráficas entre la diversificación/especialización del conocimiento tecnológico universitario y la producción de patentes universitarias en períodos posteriores, permite obtener una visión preliminar de cómo pueden afectar el patrón de diversificación y especialización existente en la región a la generación de nueva tecnología universitaria.

Para calcular las correlaciones y los gráficos de dispersión se han considerado las regiones que tienen cinco o más patentes (111 regiones). Para identificar el lapso de tiempo necesario para que una estructura diversificada/especializada tenga efectos en la producción de nuevas patentes se han utilizado varios retardos temporales (1, 2 y 3 años).

\footnotetext{
${ }^{1}$ La correspondencia entre niveles tecnológicos y los 44 sectores industriales aparecen en el Anexo I.
} 
Tabla 1

Coeficientes de correlación entre la producción de patentes y las variables de diversificación/especialización en alta, media y baja tecnología (Regiones con cinco o más patentes)

\begin{tabular}{|l|c|c|}
\hline $\begin{array}{c}\text { Variables } \\
\text { divers/especializ }\end{array}$ & $\begin{array}{c}\text { Patentes (en In) } \\
\text { Coef. Corr. }\end{array}$ & N $^{\circ}$ Obs \\
\hline IElag & $0.5694^{*}$ & 260 \\
\hline IElag2 & $0.5461^{*}$ & 226 \\
\hline IElag3 & $0.4719^{*}$ & 188 \\
\hline NVTRATlag & $0.1447^{*}$ & 260 \\
\hline NVTRATlag2 & $0.1465^{*}$ & 226 \\
\hline NVTRATlag3 & 0.1197 & 188 \\
\hline NVTRMTlag & -0.0311 & 260 \\
\hline NVTRMTlag2 & -0.0551 & 226 \\
\hline NVTRMTlag3 & -0.0411 & 188 \\
\hline NVTRBTlag & $0.1550^{*}$ & 260 \\
\hline NVTRBTlag2 & $0.1771^{*}$ & 226 \\
\hline NVTRBTlag3 & 0.1109 & 188 \\
\hline
\end{tabular}

* Significativas al 5\%.

a Variables:

- patente: Logaritmo del número de patentes en cada región.

- IElag, IElag 2, IElag 3: Índice de entropía regional con un desfase de 1, 2 y 3 años con respecto a la producción de patentes.

- NVTRATlag, NVTRATlag2, NVTRATlag3: Coeficiente de especialización regional en alta tecnología con un desfase de 1, 2, y 3 años con respecto a la producción de patentes.

- NVTRMTlag, NVTRMTlag2, NVTRMTlag3: Coeficiente de especialización regional en media tecnología con un desfase de 1, 2, y 3 años con respecto a la producción de patentes.

- NVTRBTlag, NVTRBTlag2, NVTRBTlag3: Coeficiente de especialización regional en baja tecnología con un desfase de 1, 2, y 3 años con respecto a la producción de patentes.

Fuente: Elaboración propia.

La Tabla 1 presenta los coeficientes de correlación simple. Los datos muestran que la producción de patentes universitarias está significativamente correlacionada con el nivel de entropía: a mayor nivel de diversificación tecnológica más patentes. Las cifras muestran que uno o dos años de retardo parece ser el lapso de tiempo durante el cual estas dos variables presentan las correlaciones más altas. Correlaciones significativas aparecen también entre el número de patentes en cada región y el nivel de especialización en alta y baja tecnología.

El análisis gráfico (Figuras 1 a 4) ayuda a aclarar la relación entre las variables que capturan la diversificación o la especialización con un desfase de dos 
años y la producción de patentes ${ }^{2}$.

La Figura 1 presenta para cada año una clara relación positiva entre la producción de patentes (en logaritmos y regiones con cinco o más patentes) y el índice de diversificación de entropía.

Figura 1

Relación entre la producción de patentes y la diversificación regional de patentes

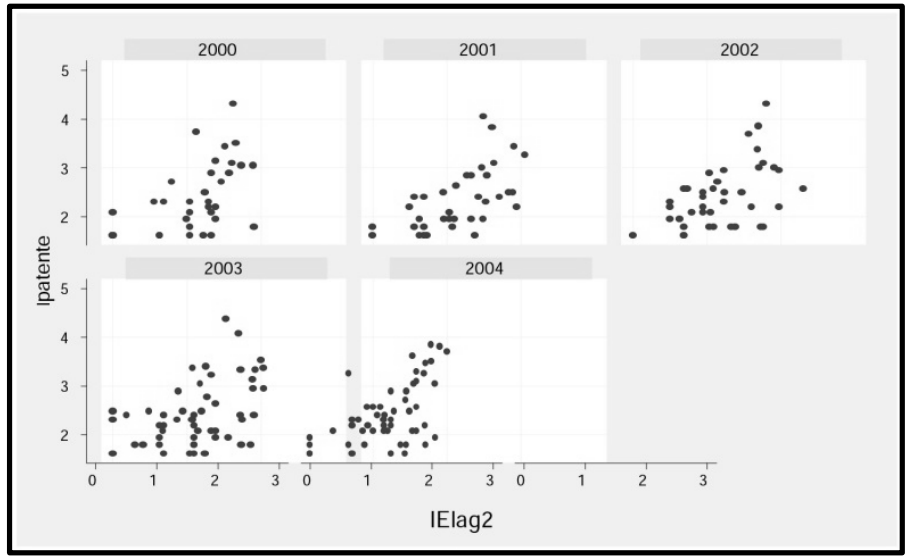

Fuente: Elaboración propia.

Figura 2

Relación entre la producción de patentes y la especialización regional en alta tecnología

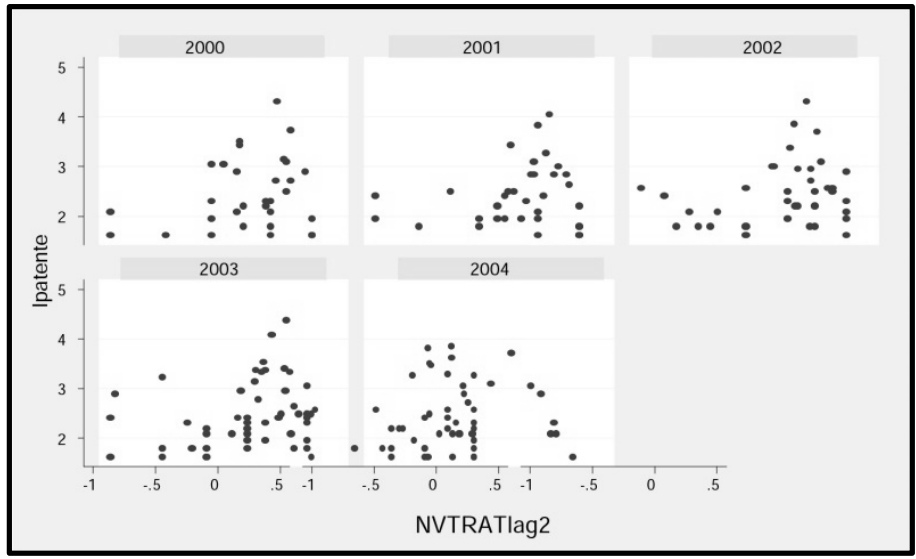

Fuente: Elaboración propia.

\footnotetext{
${ }^{2}$ Los gráficos correspondientes a los años 1998 y 1999 no se presentan debido al desfase de dos años de los índices de entropía y de especialización.
} 
Figura 3

Relación entre la producción de patentes y la especialización regional en media tecnología

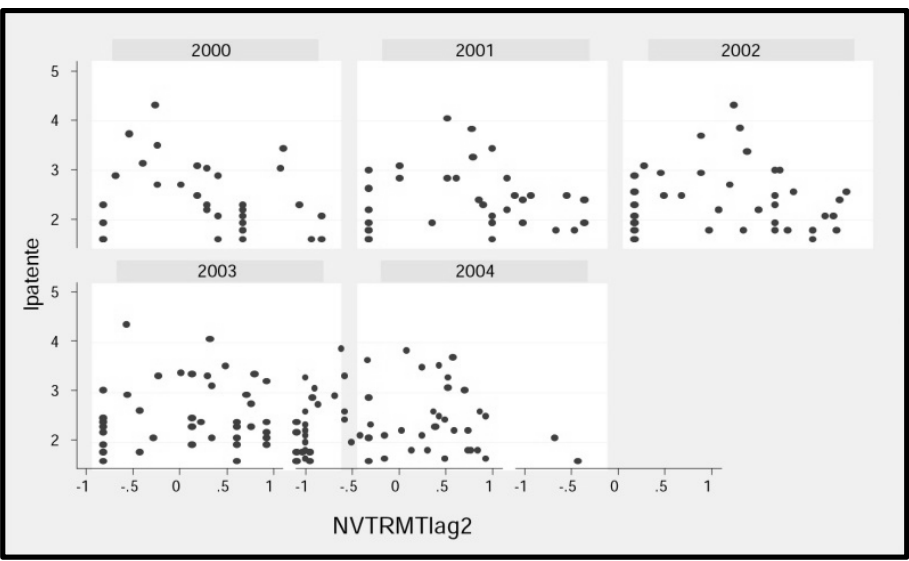

Fuente: Elaboración propia.

Figura 4

Relación entre la producción de patentes y la especialización regional en baja tecnología

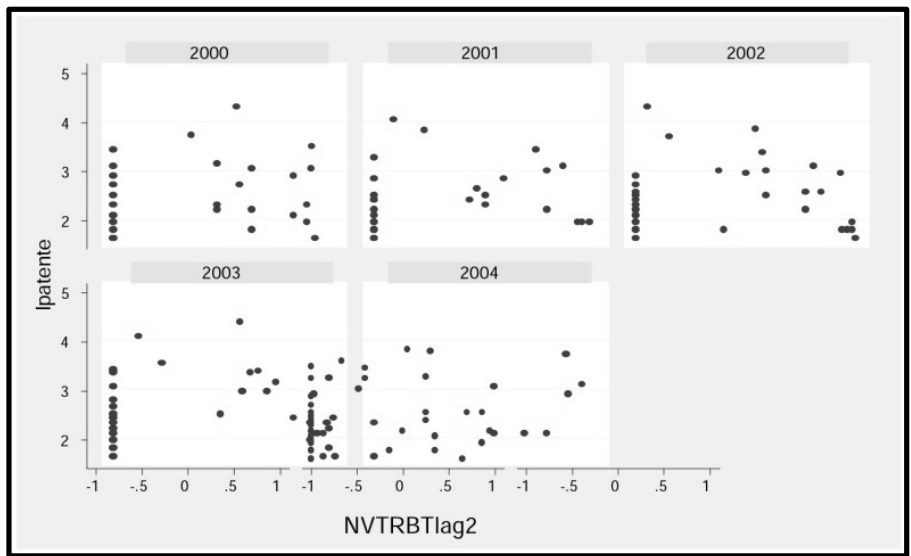

Fuente: Elaboración propia.

La Figura 2 muestra una relación positiva entre la producción de patentes y el índice de especialización en alta tecnología pero con un alto componente heterocedástico; la producción de patentes es mayor cuanto mayor es la especialización pero también crece la varianza de la producción de patentes. Las Figuras 3 y 4 recogen las relaciones entre la especialización en media y baja 
tecnología, respectivamente, con la producción de patentes mostrando que en estos casos no existen claros patrones de comportamiento.

\section{METODOLOGÍA}

Esta sección tiene por objeto establecer un modelo para evaluar la importancia de los patrones regionales de especialización/diversificación del conocimiento tecnológico universitario (junto con otras variables de control) en la producción de este tipo de conocimiento en periodos sucesivos.

\subsection{Modelo teórico}

Para analizar la relación entre la producción de conocimiento tecnológico universitario y su especialización/diversificación regional se utilizan como variables explicativas la especialización y diversificación tecnológica cuantificada por los coeficientes NVTR y el índice de entropía IE. Dado que NVTR y IE no son los únicos factores determinantes de la producción de patentes, para llegar a una especificación más adecuada se utilizan como variables adicionales otros elementos particularmente relevantes como el gasto universitario en $\mathrm{I}+\mathrm{D}$ regionalizado y los efectos temporales:

$$
P_{r t}=f\left(I E_{r t-\mu}, N V T R_{r t-\mu}, H E R D_{r t-\mu}, \text { year }_{t}, u_{r t}\right)
$$

donde $\mathrm{P}$ es la producción de nuevo conocimiento tecnológico en las universidades de la región $r$ (patentes), IE es el índice de entropía para cada región con un retardo temporal de $\mu$ años, y NVTR es el índice normalizado de la ventaja tecnológica revelada para cada región. Las variables NVTR y IE recogen respectivamente, los potenciales desbordamientos tecnológicos de la especialización y diversificación de las actividades tecnológicas de las universidades en la producción de patentes (efectos spillovers). El modelo incluye además a la principal variable para determinar $\mathrm{P}$, que es el gasto regional universitario en I+D (HERD). Los efectos temporales son recogidos a través de diversas variables binarias. Por último, "u" es el término de error.

Todas las variables explicativas se han retardado $\mu$ años debido a que se necesita tiempo para que éstas conduzcan a un resultado en forma de patente. El modelo resultante es una "función de producción de conocimiento" (Griliches, 1979) pero modificada para considerar los posibles efectos desbordamiento del conocimiento de una estrategia de especialización/diversificación tecnológica regional sobre la producción de nuevas patentes universitarias.

\subsection{Modelo empírico y Variables}

La función empírica para estimar el modelo anterior tomaría la siguiente forma: 


$$
\ln (\text { patentes })_{r t}=\alpha+\beta I E_{r t-2}+\sum_{j=1}^{j=3} v_{j} N V T R_{j r t-2}, \emptyset \ln \left(H E R D_{r t-2}\right)+\sum_{t=2001}^{t=2004} \lambda_{t} y_{e a r_{r t}}+u_{r t}
$$

donde la variable dependiente es el logaritmo del número de patentes universitarias en cada región, $r$ representa la región, j el sector (alta, media o baja tecnología) y t los años.

Las variables explicativas con dos años de retardo son las siguientes:

- Índice de entropía (IE) calculado para un máximo de 44 sectores en cada región. La diversificación del conocimiento tecnológico generado en las universidades de una región se mide a través del índice de entropía.

- Índice de ventaja tecnológica revelada normalizado (NVTR) calculado para un máximo de 44 sectores en cada región y tres niveles tecnológicos (alta, media y baja tecnología).

- Gasto regional del sector de la Educación Superior en I+D (HERD). Esta variable recoge el gasto de la regiones europeas en I+D para el sector de Educación Superior en millones de PPA a precios de 2000. Los datos proceden de Eurostat e incluyen tanto los fondos generales como un conjunto de fondos externos con los que cuenta la Educación Superior para financiar esta actividad ${ }^{3}$.

- Efectos temporales (year). Se incluyen cuatro variables binarias para considerar los efectos temporales.

Esta regresión se podría estimar utilizando el procedimiento estándar para datos de panel con efectos fijos o aleatorios. Sin embargo, este procedimiento se descartó por dos razones. En primer lugar, la variable HERD no está disponible para todas las regiones o para todos los años; la falta de datos conduce a un panel fuertemente desequilibrado que impide la aplicación de técnicas de panel por lo que se adoptó la solución de agrupar los datos teniendo en cuenta las variables ficticias que recogen los efectos temporales.

En segundo lugar, la inclusión en la función de producción de conocimiento de NVTR y IE es un aspecto novedoso que requiere cambios en la función empírica para estimar el modelo. Las estimaciones de NVTR y IE tienen sentido cuando la región cuenta con un número razonable de patentes. Para calcular estos índices e incluirlos como variables explicativas, se introdujo un proceso de selección de la muestra. Si se considera que para obtener un valor razonable de esos indicadores el número de patentes en una región debe superar un número "c", la variable dependiente observada queda truncada a la izquierda. En nuestro modelo se han considerado las regiones que tienen al menos cinco patentes. En este caso, la estimación por MCO (pooled) proporciona buenos

\footnotetext{
${ }^{3}$ Ver detalles en Frascati Manual, OECD (2002).
} 
resultados para la inferencia sobre los efectos de las variables IE y NVTR. No obstante, se realiza la estimación de modelos truncados en el análisis de robustez con el fin de obtener un mejor coeficiente para la variable HERD y en su caso, confirmar los resultados del modelo base.

La definición de todas las variables de los modelos estimados se incluye en la Tabla 2.

Tabla 2

Variables incluidas en los modelos empíricos para explicar la producción de conocimiento tecnológico universitario

\begin{tabular}{|l|l|}
\hline \multicolumn{1}{|c|}{ Variables $^{\text {a }}$} & \multicolumn{1}{c|}{ Definición } \\
\hline Ipatente & Logaritmo del número de patentes en cada región \\
\hline LHERD & $\begin{array}{l}\text { Logaritmo del gasto regional de la educación superior en I+D (HERD) (en millones de } \\
\text { PPA a precios de 2000) }\end{array}$ \\
\hline IE & Índice de entropía calculado para un máximo de 44 sectores en cada región \\
\hline $\begin{array}{l}\text { NVTRAT, } \\
\text { NVTRMT, } \\
\text { NVTRBT }\end{array}$ & $\begin{array}{l}\text { Índice normalizado de ventaja tecnológica revelada calculado para tres niveles } \\
\text { tecnológicos (Alta, Media y Baja tecnología) en cada región }\end{array}$ \\
\hline $\begin{array}{l}\text { year01, } \\
\text { year02, } \\
\text { year03, } \\
\text { year04 }\end{array}$ & Variables ficticias que recogen los efectos temporales (año base: 2000) \\
\hline
\end{tabular}

${ }^{a}$ Todas las variables para regiones con cinco o más patentes. Todas las variables explicativas con dos años de retardo.

Fuente: Elaboración propia.

\section{RESULTADOS}

En esta sección se presentan primero los resultados obtenidos en los tres modelos estimados para analizar la relevancia de las variables que recogen la diversificación/especialización del conocimiento tecnológico para explicar la producción de patentes universitarias. En una segunda subsección se incluye un estudio de robustez con varios modelos adicionales.

\subsection{Efecto de la diversificación/especialización sobre la producción de conocimiento tecnológico universitario}

La Tabla 3 muestra los principales estadísticos descriptivos para todas las variables de los modelos estimados. La Tabla 4 presenta la estimación por MCO para los tres modelos que explican los efectos de la diversificación/especialización en la producción de patentes. Dada la estructura heterocedástica de la varianza de varios regresores (véanse los gráficos presentados anteriormente), se aplicó la corrección Eicker-White a todos los modelos para obtener los valores robustos de los errores estándar. Se han incluido las variables de 
especialización en alta, media y baja tecnología en modelos separados porque una región, por lo general, se especializa en un tipo de tecnología y ocasionalmente en dos; en todo caso, cuando se incluyen ambas variables (por ejemplo, la especialización en alta y media tecnología, o en alta y baja tecnología) aparecen problemas de multicolinealidad causados por una fuerte correlación negativa entre estos pares de variables.

Tabla 3

Estadísticos descriptivos

\begin{tabular}{|c|c|c|c|c|}
\hline Variable & Media & Dev. Est. & Min & Max \\
\hline Ipatente & 2,3930 & 0,6646 & 1,6094 & 4,3695 \\
\hline LHERDlag2 & 5,5895 & 0,7668 & 3,9502 & 7,6171 \\
\hline IElag2 & 1,1453 & 0,5759 & 0,0000 & 2,3026 \\
\hline NVTRATlag2 & $-0,0860$ & 0,3719 & $-1,0000$ & 0,3615 \\
\hline NVTRMTlag2 & $-0,1467$ & 0,6648 & $-1,0000$ & 0,9272 \\
\hline NVTRBTlag2 & $-0,4017$ & 0,7837 & $-1,0000$ & 0,9926 \\
\hline$N^{\circ}$ obs & 121 & & & \\
\hline
\end{tabular}

Fuente: Elaboración propia.

Tabla 4

Modelo de datos fusionados para regiones de cinco o más patentes

\begin{tabular}{|c|c|c|c|c|c|c|c|c|c|}
\hline \multicolumn{10}{|c|}{ Var Dep: Ipatente } \\
\hline & \multicolumn{3}{|c|}{ (1) } & \multicolumn{3}{|c|}{ (2) } & \multicolumn{3}{|c|}{ (3) } \\
\hline & Coef. & & Err. Est. & Coef. & & Err. Est. & Coef. & & Err. Est. \\
\hline LHERDlag2 & 0,2827 & 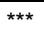 & 0,0668 & 0,2887 & $* * *$ & 0,0667 & 0,3099 & $* * *$ & 0,0682 \\
\hline IElag2 & 0,4929 & $\star \star \star *$ & 0,0772 & 0,5304 & $* * *$ & 0,0785 & 0,5285 & $* * *$ & 0,0822 \\
\hline NVTRATlag2 & 0,3108 & ** & 0,1309 & & & & & & \\
\hline NVTRMTlag2 & & & & $-0,1511$ & $* *$ & 0,0688 & & & \\
\hline NVTRBTlag2 & & & & & & & $-0,1114$ & * & 0,0613 \\
\hline Year $_{2001}$ & $-0,0029$ & & 0,1718 & $-0,0179$ & & 0,1703 & $-0,0200$ & & 0,1501 \\
\hline Year $_{2002}$ & 0,1266 & & 0,1574 & 0,1064 & & 0,1580 & 0,1594 & & 0,1466 \\
\hline Year $_{2003}$ & 0,3293 & $\star *$ & 0,1620 & 0,3043 & * & 0,1602 & 0,3477 & ** & 0,1479 \\
\hline Year $_{2004}$ & 0,3616 & $\star \star$ & 0,1702 & 0,3372 & $* *$ & 0,1695 & 0,3598 & ** & 0,1603 \\
\hline Cons & 0,0851 & & 0,4049 & $-0,0216$ & & 0,4030 & $-0,1924$ & & 0,4108 \\
\hline $\mathrm{N}^{\circ}$ obs & 121 & & & 121 & & & 121 & & \\
\hline$F(7,113)$ & 13,09 & 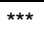 & & 14,33 & *** & & 14,10 & 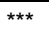 & \\
\hline Prob $>F$ & 0,0000 & & & 0,0000 & & & 0,0000 & & \\
\hline R-squared & 0,4812 & & & 0,4730 & & & 0,4667 & & \\
\hline Root MSE & 0,4933 & & & 0,4972 & & & 0,5002 & & \\
\hline
\end{tabular}

*** Sign. al 1\%; ** Sign. al 5\%; * Sign. al 10\%.

Fuente: Elaboración propia. 
Los resultados muestran que la variable IE con un retardo de dos años (IElag2) es positiva y altamente significativa en todos los modelos, apoyando la hipótesis de que la diversificación tecnológica explica la producción de patentes universitarias en periodos posteriores de manera que, cuanto mayor es la diversificación del conocimiento tecnológico universitario generado en las regiones, mayor es el número de patentes producidas en el futuro. Las regiones de Outer London (GB), Dresden (DE), Hampshire and Isle of Wight (GB), Herefordshire, Worcestershire and Warwickshire (GB) y Stuttgart (DE) se encuentran entre las que presentan mayor diversificación tecnológica. Las tres variables de especialización tecnológica son también importantes pero con significados muy diferentes. El coeficiente de la variable NVTRATlag2 (especialización en alta tecnología con un retardo de dos años) es positivo y significativo al $5 \%$, por lo tanto, aumentar la especialización en sectores de alta tecnología se traduciría en más patentes en los períodos posteriores, mientras que aumentar la especialización en media o baja tecnología produciría el efecto opuesto. Kassel (DE), Border, Midland and Western (IR), Pohjois-Suomi (FI), Picardie (FR) y NoordHolland (NL) son algunas de las regiones con mayor especialización en sectores de alta tecnología.

Los modelos muestran que una estrategia de diversificación en la producción de conocimiento tecnológico universitario favorece la generación de nuevo conocimiento tecnológico y al mismo tiempo la especialización es una buena estrategia pero sólo en sectores de alta tecnología. Otras variables en los modelos son LHERDlag2 (logaritmo del gasto regional de la educación superior en I+D) y las variables ficticias que recogen los efectos temporales. Con respecto a la variable LHERDlag2, se obtienen coeficientes significativos con elasticidades de alrededor de 0,3 ; no obstante, hay que tener en cuenta que estas elasticidades son solo justificables para las regiones que producen cinco o más patentes.

\subsection{Robustez}

Para evaluar la robustez de los resultados, se han analizado los posibles cambios cuando se aumenta el número de patentes de cada región. Se han reproducido los modelos anteriores para regiones con seis o más patentes, siete o más, y así sucesivamente. Los resultados de las estimaciones de estos nuevos modelos no difieren sustancialmente de los modelos (1) a (3) de la Tabla 4; los mismos coeficientes siguen siendo significativos. La Tabla 5 muestra los resultados de las estimaciones para regiones con diez o más patentes.

Hasta ahora el interés se ha centrado en los efectos de la especialización (NVTR) y la diversificación (IE) en la producción de patentes universitarias, pero los modelos estimados incluyen otra variable relevante: los gastos regionales de educación superior en I + D (LHERDlag2). De acuerdo con las estima- 
ciones realizadas, esta variable es altamente significativa para explicar la producción de patentes universitarias cuando la región tiene cinco o más patentes (seis, siete,..., o diez o más en los últimos modelos) con elasticidades alrededor de 0,30 . Sin embargo, los gastos regionales de educación superior en I + D deben explicar la producción de las patentes universitarias en todas las regiones no sólo en aquellas en las que el número de patentes universitarias es mayor que "c". Esto significa que si nos centramos en esta variable la especificación debe ser tratada como truncada a la izquierda.

Tabla 5

Modelo de datos fusionados para regiones de 10 ó más patentes

\begin{tabular}{|c|c|c|c|c|c|c|c|c|c|}
\hline \multicolumn{10}{|c|}{ Var Dep: Ipatente } \\
\hline & \multicolumn{3}{|c|}{ (4) } & \multicolumn{3}{|c|}{ (5) } & \multicolumn{3}{|c|}{ (6) } \\
\hline & Coef. & & Err. Est. & Coef. & & Err. Est. & Coef. & & Err. Est. \\
\hline LHERDlag2 & 0.2980 & $* \star \star$ & 0.0722 & 0.2996 & $* * *$ & 0.0735 & 0.3008 & $* * *$ & 0.0720 \\
\hline IElag2 & 0.3643 & *** & 0.1001 & 0.3983 & $* * *$ & 0.0938 & 0.3917 & $* * *$ & 0.1027 \\
\hline NVTRATlag2 & 0.2595 & ** & 0.1098 & & & & & & \\
\hline NVTRMTlag2 & & & & -0.1563 & $\star \star$ & 0.0763 & & & \\
\hline NVTRBTlag2 & & & & & & & -0.0670 & & 0.0653 \\
\hline Year $_{2001}$ & 0.1013 & & 0.2410 & 0.0566 & & 0.2471 & 0.0609 & & 0.2115 \\
\hline Year $_{2002}$ & 0.3235 & & 0.2580 & 0.2613 & & 0.2606 & 0.3617 & & 0.2472 \\
\hline Year $_{2003}$ & 0.4168 & * & 0.2443 & 0.3525 & & 0.2414 & 0.4352 & * & 0.2295 \\
\hline Year $_{2004}$ & 0.3064 & & 0.2371 & 0.2279 & & 0.2352 & 0.3114 & & 0.2219 \\
\hline Cons & 0.4049 & & 0.4940 & 0.3725 & & 0.5028 & 0.3073 & & 0.4940 \\
\hline $\mathrm{N}^{\circ}$ obs & 59 & & & 59 & & & 59 & & \\
\hline$F(7,113)$ & 10.07 & $* \star \star$ & & 9.86 & $* * *$ & & 8.58 & $* * *$ & \\
\hline R-squared & 0.5340 & & & 0.5324 & & & 0.5120 & & \\
\hline Root MSE & 0.3766 & & & 0.3772 & & & 0.3854 & & \\
\hline
\end{tabular}

*** Sign. al 1\%; ** Sign. al 5\%; * Sign. al 10\%.

Fuente: Elaboración propia.

El procedimiento $\mathrm{MCO}$ no proporciona estimaciones correctas de este coeficiente (la estimación es sesgada e inconsistente y lo mismo ocurre para la estimación de la varianza del error) por lo que se ha aplicado un estimador QML (cuasi máxima verosimilitud) (Tabla 6). Las elasticidades están comprendidas entre 0,43 y 0,50 mayores que en los modelos anteriores. 
Tabla 6

Resultados del modelo truncado (QML). Regiones con cinco o más patentes

\begin{tabular}{|c|c|c|c|c|c|c|c|c|c|}
\hline \multicolumn{10}{|c|}{ Dep Var: Ipatente (truncada a la izquierda para regiones con 5 o más patentes) } \\
\hline & \multicolumn{3}{|c|}{ (7) } & \multicolumn{3}{|c|}{ (8) } & \multicolumn{3}{|c|}{ (9) } \\
\hline & Coef. & & Err. Est. & Coef. & & Err. Est. & Coef. & & Err. Est. \\
\hline LHERDlag2 & 0,4380 & *** & 0,1057 & 0,4550 & $* * *$ & 0,1059 & 0,5081 & $* * *$ & 0,1164 \\
\hline IElag2 & 1,0785 & *** & 0,1796 & 1,1638 & $* * *$ & 0,1964 & 1,1784 & $* * *$ & 0,2090 \\
\hline NVTRATlag2 & 0,8595 & 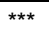 & 0,3610 & & & & & & \\
\hline NVTRMTlag2 & & & & $-0,3561$ & $* * *$ & 0,1540 & & & \\
\hline NVTRBTlag2 & & & & & & & $-0,1889$ & 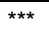 & 0,1144 \\
\hline Year $_{2001}$ & 0,2800 & & 0,3930 & 0,2128 & & 0,4108 & 0,2960 & & 0,3993 \\
\hline Year $_{2002}$ & 0,5236 & & 0,3563 & 0,4566 & & 0,3699 & 0,5784 & & 0,3764 \\
\hline Year $_{2003}$ & 0,7489 & $* *$ & 0,3592 & 0,6759 & * & 0,3680 & 0,8139 & * & 0,3748 \\
\hline Year $_{2004}$ & 0,7195 & * & 0,3732 & 0,6413 & * & 0,3858 & 0,7210 & * & 0,3950 \\
\hline Cons & $-2,2994$ & $* * *$ & 0,8129 & $-2,5644$ & $* * *$ & 0,8459 & $-3,0276$ & $* * *$ & 0,9304 \\
\hline Sigma & 0,7046 & $* * *$ & 0,0733 & 0,7186 & & 0,0756 & 0,7324 & & 0,0752 \\
\hline $\mathrm{N}^{\circ} \mathrm{Obs}$ & 142 & & & 142 & & & 142 & & \\
\hline Wald chi2(7) & 65,63 & $* * *$ & & 61,06 & & & 57,06 & & \\
\hline Log pseudoLh. & $-75,081$ & & & $-76,94$ & & & $-78,82$ & & \\
\hline
\end{tabular}

*** Sign. al 1\%; ** Sign. al 5\%; * Sign. al 10\%.

Fuente: Elaboración propia.

\section{CONCLUSIONES}

Las universidades desempeñan en las regiones un papel relevante en la generación de conocimiento tecnológico. Sin embargo, la literatura empírica ha estado tradicionalmente centrada en los efectos de los resultados de las actividades de las universidades en las variables relevantes para las economías regionales (innovación, creación de empresas, los resultados empresariales, etc.) pero muy poco se sabe acerca de las causas de la mayor producción de tecnología por las instituciones universitarias. En este trabajo, se ha tratado de llenar este hueco con una investigación desde la perspectiva de la oferta: la producción de conocimiento tecnológico medida por las patentes generadas por las universidades en las regiones europeas. En particular, el interés se ha centrado en analizar si la diversificación o la especialización tecnológica de las universidades podrían conducir a la producción de más resultados tecnológicos en el futuro. Desde un punto de vista político, este debate es relevante porque promover una u otra estrategia puede dar lugar a resultados diferentes.

Los resultados obtenidos a partir de una función de producción de conocimiento para las regiones europeas, se pueden resumir en los siguientes:

- El coeficiente del índice de entropía con un retardo de dos años es positivo y altamente significativo. Esta conclusión avala la idea de que la 
diversificación del conocimiento tecnológico en un período se traduciría en un aumento de los resultados tecnológicos en los períodos siguientes.

- El coeficiente de especialización regional en alta tecnología (con un retardo de dos años) es positivo y significativamente relacionado con la producción de patentes universitarias, mientras que la especialización regional en tecnología media y baja tiene un efecto negativo en el número de patentes en los períodos siguientes.

- El coeficiente del gasto regional de educación superior en I + D (incluido como una variable de control) es significativo en todos los modelos, con elasticidades alrededor de 0,2 - 0,3 para las regiones con cinco o más patentes y de 0,5 para el conjunto de la población. Similares resultados se obtuvieron en estudios previos en otros contextos (por ejemplo, Payne and Siow, 2003); sin embargo, este resultado debe considerarse con cautela debido a los problemas en la medición de esta variable.

Desde un punto de vista político (regional o nacional) estos resultados ofrecen algunas pistas sobre las estrategias a seguir con el fin de promover la producción de tecnología en las universidades europeas. Dos ideas principales surgen al respecto: en primer lugar, fomentar la diversificación apoyando proyectos con resultados en múltiples sectores y en segundo lugar, estimular la producción de conocimiento tecnológico universitario sólo en los sectores de alta tecnología. Téngase en cuenta que diversificación y especialización no son conceptos necesariamente excluyentes entre sí. Una economía regional puede tener unas pocas industrias especializadas y al mismo tiempo ser diversa; la misma idea es válida para el conocimiento tecnológico universitario producido en las regiones.

\section{REFERENCIAS BIBLIOGRÁFICAS}

ACOSTA, M., CORONADO, D., LEÓN, D. y MARTÍNEZ, M.A. (2009): "The production of university technological knowledge in European regions: evidence from patent data”. Regional Studies, 43(9), pp. 1167-1181.

ACS, Z.J., ANSELIN, L., y VARGA, A. (2002): "Patents and innovation counts as measures of regional production of new knowledge". Research Policy, 31(7), pp. 1069-1085.

AUDRETSCH, D.B. y LEHMANN, E.E. (2005): "Does the knowledge spillover theory of entrepreneurship hold for regions?”, Research Policy, 34, pp. 1191-1202.

AZAGRA-CARO, J.M., FERNANDEZ-DE-LUCIO, I. y GUTIERREZ-GRACIA, A. (2003): "University patents: output and input indicators... of what?" Research Evaluation, 12 (1), pp. 5-16. 
AZAGRA-CARO, J.M., YEGROS-YEGROS, A. y ARCHONTAKIS, F. (2006): "What do university patent routes indicate at regional level?". Scientometrics, 66 (1), pp. 219230.

BALASSA, B. (1965): "Trade liberalization and "revealed" comparative advantage". The Manchester School of Economic and Social Studies, 32, pp. 99-123.

BALDINI, N., GRIMALDI, R. y SOBRERO, M. (2006): "Institutional changes and the commercialization of academic knowledge: A study of Italian universities' patenting activities between 1965 and 2002". Research Policy, 35 (4), pp. 518-532.

BAPTISTA, R. y SWANN, P. (1998): "Do firms in clusters innovate more?". Research Policy, 27, pp. 525-540.

BEAUDRY, C. y BRESCHI, S. (2003): "Are firms in clusters really more innovative?". Economics of Innovation and New Technology, 12(4), pp. 325-342

BEAUDRY, C. y SCHIFFAUEROVA, A (2009): "Who's right, Marshall or Jacobs?. The localization versus urbanization debate". Research Policy, 38, pp. 318-337.

BERCOVITZ, J. y FELDMANN, M. (2006): "Entpreprenerial universities and technology transfer: a conceptual framework for understanding knowledge-based economic development". Journal of Technology Transfer, 31 (1), pp. 175-188.

CARLINO, G.A., CHATTERJEE, S. y HUNT, R.M. (2007): "Urban density and the rate of invention". Journal of Urban Economics, 61, pp. 389-419.

DJOKOVIC, D. y SOUITARIS, V. (2008): "Spinouts from academic institutions: a literature review with suggestions for further research". Journal of Technology Transfer, 33 (3), pp. 225-247.

EJERMO, O. (2005): “Technological Diversity and Jacobs' Externality Hypothesis Revisited". Growth and Change, 36 (2), pp. 167-195.

ETZKOWITZ, H. y KLOFSTEN, M. (2005): "The innovating region: toward a theory of knowledge-based regional development". R\&D Management, 35 (3), pp. 243-255.

ETZKOWITZ, H. y ZHOU, C. (2006): "Triple helix twins: innovation and sustainability". Science and Public Policy, 33, pp. 77-83.

ETZKOWITZ, H., RANGA, M., BENNER, M., GUARANYS, L., MACULAN, A.M. y KNELLER, R. (2008): "Pathways to the entrepreneurial university: towards a global convergence". Science and Public Policy, 35 (9), pp. 681-695.

FELDMAN, M.P. y AUDRETSCH, D.B. (1999): "Innovation in cities: science-based diversity, specialization and localized competition”. European Economic Review 43, pp. 409-429.

FRITSCH, M. y SLAVTCHEV, V. (2007): “Universities and innovation in space”. Industry and Innovation, 14 (2), pp. 201-218.

GEUNA, A. y NESTA, L.J.J. (2006): "University patenting and its effects on academic research: the emerging European evidence”. Research Policy, 35 (6), pp. 790-807.

GLAESER, E.L., KALLAL, H.D., SCHEINKMAN, J.A. y SHLEIFER, A. (1992): "Growth in cities". Journal of Political Economy, 100 (6), pp. 1126-1152.

GREUNZ, L. (2003): "The impact of industrial specialisation and diversity on innovation". Brussels Economic Review-Cahiers Economiques de Bruxelles, 46 (3), pp. 11-36.

GRILICHES, Z. (1979): "Issues in assessing the contribution of research and development to productivity growth". Bell Journal of Economics, 10, pp. 92-116. 
GRUPP, H. (1994): "The measurement of technical performance of innovations by technometrics and its impact on established technology indicators". Research Policy, 23, pp. 175-193.

JACOBS, J. (1969): The Economy of Cities. New York: Random House.

KELLY, M. y HAGEMAN, A. (1999): "Marshallian externalities in innovation". Journal of Economic Growth, 4, (1), pp. 39-54.

MORENO, R., PACI, R. y USAI, S. (2006): "Innovation Clusters in the European Regions". European Planning Studies, 14 (9), pp.1235-1263.

O'HUALLACHÁIN, B. y LEE, D-S. (2011): "Technological Specialization and Variety in Urban Invention". Regional Studies, 45 (1), pp. 67-88.

O'SHEA, R.P., CHUGH, H. y ALLEN, T.J. (2008): "Determinants and consequences of university spinoff activity: a conceptual framework". Journal of Technology Transfer, 33, pp. 653-667.

OECD (2002). Frascati Manual 2002: Proposed Standard Practice for Surveys on Research and Experimental Development. Paris: OECD.

PACI, R. y USAI, S.(1999): "Externalities, knowledge spillovers and the spatial distribution of innovation". GeoJournal, 49, pp. 381-390.

PAYNE, A. y SIOW, A. (2003): "Does federal research funding increase university research output?". Advances in Economic Analysis \& Policy, 3 (1), pp.1018.

SARAGOSSI S. y VAN POTTELSBERGHE B. D. L. P. (2003):"What patent data reveal about universities: the case of Belgium". Journal of Technology Transfer 28, pp. 4751.

SCHMOCH, U., LAVILLE, F., PATEL, P. y FRIETSCH, R. (2003): Linking technology areas to industrial sectors. Final Report to the European Commission, DG Research.

SHEFER, D. y FRENKEL, A. (1998): "Local milieu and innovations: Some empirical results". Annals of Regional Science, 32, pp. 185-200.

VARGA, A. (1998): University Research and Regional Innovation: A Spatial Econometric Analysis of Academic Technology Transfers. Boston: Kluwer Academic Publishers.

WOODWARD, D., FIGUEIREDO, O. y GUIMARAES, P. (2006): "Beyond the Silicon Valley: university R\&D and high-technology location". Journal of Urban Economics, 60 (1), pp. 15-32.

YUSOF, M. y JAIN, K.K. (2010): "Categories of university-level entrepreneurship: a literature survey". International Entrepreneurship and Management Journal, 6, pp. 81-96. 
ANEXO I

Correspondencia entre los 44 sectores y nivel tecnológico

\begin{tabular}{|c|c|c|}
\hline $\begin{array}{c}\text { Nivel } \\
\text { tecnológico }\end{array}$ & $\begin{array}{c}\mathbf{N}^{\circ} \\
\text { sector }\end{array}$ & Descripción códigos NACE \\
\hline \multirow{10}{*}{$\begin{array}{c}\text { Alta } \\
\text { tecnología }\end{array}$} & 13 & Productos farmacéuticos \\
\hline & 28 & Máquinas de oficina y equipos informáticos \\
\hline & 34 & Componentes electrónicos \\
\hline & 35 & Transmisores de radio y televisión; telecomunicaciones \\
\hline & 36 & Aparatos recepción, grabación y reproducción de sonido e imagen \\
\hline & 37 & Aparatos médicos \\
\hline & 38 & Instrumentos de medida \\
\hline & 39 & Equipo de control de procesos industriales \\
\hline & 40 & Instrumentos de óptica y de equipo fotográfico \\
\hline & 41 & Fabricación de relojes \\
\hline \multirow{26}{*}{$\begin{array}{c}\text { Media } \\
\text { tecnología }\end{array}$} & 9 & Coquerías, refino de petróleo y combustible nuclear \\
\hline & 10 & Productos químicos básicos \\
\hline & 11 & Pesticidas y otros productos agroquímicos \\
\hline & 12 & Pinturas, barnices y revestimientos similares \\
\hline & 14 & Jabones, detergentes, perfumes y productos de belleza e higiene \\
\hline & 15 & Otros productos químicos \\
\hline & 16 & Fibras artificiales \\
\hline & 17 & Productos de caucho y materias plásticas \\
\hline & 18 & Otros productos minerales no metálicos \\
\hline & 19 & Metalurgia \\
\hline & 20 & Productos metálicos \\
\hline & 21 & Máquinas, equipo y material mecánico \\
\hline & 22 & Otras máquinas, equipo y material mecánico \\
\hline & 23 & Maquinaria agraria \\
\hline & 24 & Máquinas herramienta \\
\hline & 25 & Maquinaria diversa para usos específicos \\
\hline & 26 & Armas y municiones \\
\hline & 27 & Aparatos domésticos \\
\hline & 29 & Generadores, transformadores y motores eléctricos \\
\hline & 30 & Aparatos distribución y control eléctrico; hilos y cables eléctricos \\
\hline & 31 & Acumuladores y pilas eléctricas \\
\hline & 32 & Lámparas eléctricas y aparatos de iluminación \\
\hline & 33 & Otro equipo eléctrico \\
\hline & 42 & Vehículos de motor, remolques y semirremolques \\
\hline & 43 & Otro material de transporte \\
\hline & 44 & Muebles; otras industrias manufactureras \\
\hline \multirow{8}{*}{$\begin{array}{c}\text { Baja } \\
\text { tecnología }\end{array}$} & 1 & Productos alimenticios y bebidas \\
\hline & 2 & Industria del tabaco \\
\hline & 3 & Industria textil \\
\hline & 4 & Industria de la confección y de la peletería \\
\hline & 5 & Industria del cuero y del calzado \\
\hline & 6 & Industria de la madera y del corcho \\
\hline & 7 & Industria del papel \\
\hline & 8 & Edición, artes gráficas; reproducción de soportes grabados \\
\hline
\end{tabular}

Fuente: Elaboración propia a partir de Schmoch et al. (2003) y clasificación OCDE. 
